\title{
QUAND LE DICTIONNAIRE DONNE A VOIR LA CULTURE
}

\author{
Sylvester N. OSU ${ }^{1}$ \\ Laboratoire Ligérien de Linguistique (UMR 7270), \\ Université François Rabelais, Tours (France)
}

\begin{abstract}
Résumé. L'ikwere, une langue Benue-Congo parlée au sud-est du Nigeria, est une langue à tradition orale, très peu décrite et très mal documentée. C'est donc pour contribuer à une meilleure documentation de cette langue que $\mathrm{j}$ 'ai entrepris la confection d'un dictionnaire trilingue ikwere-anglais-français. Or, ce projet de dictionnaire se veut le reflet de la culture ikwere. En prenant comme exemple quelques noms de personne et de lieu mais aussi le calendrier et le vocabulaire du mariage, cet article montre comment j'aborde des aspects de la culture ikwere dans les entrées de ce projet de dictionnaire sur lequel je travaille à l'aide du logiciel Toolbox depuis quelques années. Ainsi, je donne pour les entrées, et lorsque cela me paraît à la fois pertinent pour mieux cerner l'entrée en question, et illustratif pour la culture ikwere, des renseignements élaborés dans le champ «notes et commentaires». Ce projet de dictionnaire comprend actuellement 2360 entrées.
\end{abstract}

Mots-clés : ikwere, Nigeria, ethnolinguistique, dictionnaire plurilingue, base de données électronique

\section{Introduction}

Afin de contribuer à une meilleure documentation de la langue ikwere, ${ }^{2}$ une langue à tradition orale, très peu documentée et très peu décrite, j'ai entrepris depuis quelques années, l'élaboration d'un dictionnaire trilingue général, ${ }^{3}$ ikwere-anglais-français. Ce projet est toujours en cours actuellement.

L'objectif de cet article consiste à présenter des aspects de ce projet de dictionnaire. Plus précisément, cet article soutient que ce type de dictionnaire doit à terme, contenir des informations d'ordre culturel et spécifique afin de rendre les entrées plus faciles à cerner. Il vise également à décrire de telles informations et à montrer de quelle manière elles sont insérées dans le dictionnaire, en prenant comme exemples le nom de personne et de lieu, mais aussi le calendrier et le vocabulaire du mariage.

1 Je tiens à remercier les relecteurs anonymes ainsi que l'éditeur de Studies in African Linguistics. Leurs remarques et suggestions m'ont permis d'améliorer considérablement cet article. Je reste seul responsable de toute erreur qu'on pourrait y relever.

2 Langue SVO, selon la méthode de classification typologique, avec une morphologie verbale flexionnelle et une structure syllabique $\mathrm{CV}$, la langue ikwere [i $\mathrm{k}^{\mathrm{w}}$ éré] est parlée par un peuple qui porte le même nom dans l'Etat des Rivières (Rivers State) au sud-est du Nigeria en Afrique de l'Ouest. Elle compte environ 1,2 million de locuteurs et est l'une des sept langues igboïdes identifiées à l'heure actuelle, du groupe Benue-Congo de la branche Niger-Congo. Les autres langues du groupe igboïde sont igbo, ika, izii, ogba, ukwuani et ẹkpẹyẹ.

3 Voir Marello (1996: 37-42) pour une typologie des dictionnaires bilingues. Elle distingue (p. 37) des dictionnaires généraux plus ou moins encyclopédiques, des dictionnaires abrégés, des dictionnaires de poche, des dictionnaires spécialisés, des ouvrages sémasiologiques et des ouvrages onomasiologiques. 
Cette position est loin d'être isolée. Marello (1996 : 37) écrivait déjà : « [L]es [dictionnaires] bilingues généraux devraient contenir aussi un certain nombre de noms propres de personnes et de lieux, surtout quand ces noms propres diffèrent d'une langue à l'autre. » Et s'il est vrai, comme le dit Marello quelques lignes plus loin dans son texte, que « [b]eaucoup de [dictionnaires] bilingues n'englobent pas les noms propres dans la macrostructure principale du dictionnaire, mais les présentent dans une partie séparée, sous forme de listes, qui en donnent la graphie, mais négligent souvent la prononciation ", le projet de dictionnaire ikwere-anglais-français, lui, adopte une approche différente : il intègre ces informations directement dans la macrostructure principale. ${ }^{4}$

D'une manière générale, l'élaboration d'un dictionnaire bilingue ou multilingue pour une langue peu documentée tend à répertorier les unités de la langue source et à donner leurs équivalents dans la langue d'arrivée. Le tout est souvent bien accompagné d'exemples (allant de zéro exemple à plusieurs) qui illustrent de façon merveilleuse certains aspects de la culture en question. C'est en tout cas ce que semble suggérer Roulon-Doko (2008: 7) dans le passage suivant: « $[\ldots]$, ce dictionnaire nous renseigne sur tous les aspects de la vie de ces chasseurs cueilleurs cultivateurs qui vivent dans une savane verte dont ils connaissent aussi bien le monde animal que le monde végétal, et où ils pratiquent leurs activités quotidiennes ».

Cette approche est celle de, entre autres, Caron \& Amfani (1997), Echeruo (1998), Kropp Dakubu (1999), Sachnine (1997), Taylor (1932), Ugochukwu \& Okafor (2004), et Williamson (1972).

Ce qui caractérise le projet de dictionnaire trilingue ikwere-anglais-français, c'est qu'il ne se limite pas à une liste de mots et leurs équivalents. Il ne se limite pas non plus aux exemples qui renseignent sur tel ou tel aspect de la culture ikwere. Il tente de fournir des informations linguistiques et culturelles supplémentaires à l'utilisateur afin de lui permettre de mieux appréhender les unités de la langue.

Concernant les informations de type linguistique, ce projet de dictionnaire fournit les caractéristiques phonologiques, morphologiques, syntaxiques et sémantiques des entrées. Corollairement, il propose une caractérisation métalinguistique du fonctionnement de l'unité lorsque celle-ci est accessible. C'est-à-dire lorsque l'unité en question a fait l'objet d'une description. La caractérisation métalinguistique alors est le résultat d'une étude minutieuse et approfondie du mode de fonctionnement invariant et spécifique de l'unité à travers sa diversité d'emplois et de fonctions.

Quant aux informations de type culturel, il s'agit d'informations supplémentaires et extralinguistiques introduites lorsque le lien entre l'unité dans la langue de départ et l'équivalent proposé dans la langue d'arrivée n'est pas du tout évident. On montre ainsi, que la prise en compte du rôle de la culture dans la construction du sens des unités de la langue permet une meilleure description et donc une meilleure présentation des entrées lexicales. Dans une perspective semblable, Diki-Kidiri, Mbodj \& Edema (1997) écrivent :

4 Le lecteur pourra se reporter à Cristinoï et Nemo (2013: 126). Ces auteurs soulignent le fait que l'élaboration d'un dictionnaire pour une langue peu documentée implique un certain nombre de contraintes. De ce fait, elle se différencie de l'élaboration d'un dictionnaire standard (c'est-à-dire, l'élaboration de dictionnaires pour des langues de grande diffusion) et impose des stratégies à la fois méthodologique et lexicographique différentes. 
Cela ne nous instruit pas énormément de savoir que l'anglais «software» se dit « logiciel » en français. Ce qui est instructif, par contre, c'est de savoir comment ces mots ont été choisis [...]. Car c'est seulement dans la description analytique de l'histoire de chaque mot, l'évolution de sa forme, les glissements de ses sens, les procédés cognitifs mis en jeu que nous comprendrons comment l'homme, tant individuellement que socialement, s'approprie de nouvelles connaissances, se construit de nouveaux concepts et, finalement, évolue lui-même en rénovant sa culture. [p 95]

En ikwere ákârik ưgà et ákârìtá sont les deux manières de désigner «(la) main droite » et «(la) main gauche » respectivement. On peut reconnaître l'unité ákâ pour «main» dans les deux exemples. On peut également reconnaître $i k^{w} \dot{u} g a ̀$ dans le premier cas. Mon enquête et ma connaissance personnelle révèlent que ce dernier élément est un nom de personne. Ce qui autorise une traduction littérale comme ceci : "la main de $i k^{w} u$ úgà ». En revanche, ìtá reste un élément inconnu des gens interrogés. A quoi se réfère le ' $r$ ' dans les deux exemples ? Serait-il un élément épenthétique ? On peut considérer qu'il n'est pas nécessaire de décomposer ces unités. Mais on peut aussi penser qu'il est intéressant de savoir pourquoi dans la culture ikwere on choisit de nommer la main après (r)ik úgà ou (r)ìtá. On trouve l'exemple (1) aussi bien dans la langue ikwere que dans la langue igbo. ${ }^{5}$

(1) àtâ $\quad$ áh $^{\mathrm{w}}$ inh $^{\mathrm{w}}$ '́

B.V.mâcher asticot

Souffrir.

m̀ tà-rò-m áh ${ }^{w}{ }_{\text {ón }}^{\mathrm{w}}$ hứ k-ězí n-òhà̀ k-êm

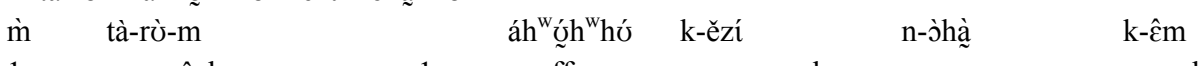

1SG B.V.mâcher-EFF-ACCORD 1SG souffrance SPEC-grandeur REL-commune SPEC-là

J'ai beaucoup souffert quand j'habitais dans ce pays-là.

(3) án ${ }^{w}{ }_{\text {ớ }}^{\mathrm{w}}{ }^{\mathrm{w}}$ ứ ó n-àtâ n-ákâ ǹzì á wêm kárí-lêm

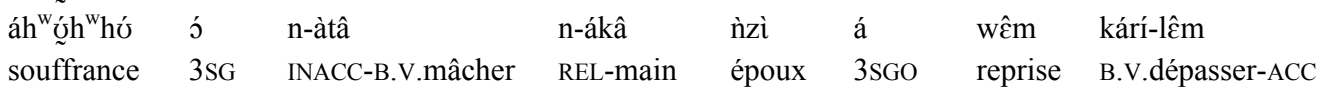

Litt. : La souffrance qu'elle subit dans les mains de son mari dépasse l'acceptable.

Elle souffre énormément chez son mari.

L'ikwere a une orthographe officielle qui présente des difficultés aux lecteurs non-initiés. Ainsi, un point souscrit à une voyelle indique le trait -ATR comme ceci : ẹ $=\varepsilon, o ̣=\rho, i ̣=I, ~ u ̣=~ v$. L'ikwere connaît en effet, un système d'harmonie vocalique selon le trait [ATR]. Sinon, $g h=y, h=x, w h=h^{w}, y n=n$; une voyelle précédée du graphème $n$ indique la nasalité, p.ex. àvnụ̂ $=$ avợ, bìsní $=$ bìsí, ìwhnérné $=$ ih $^{\mathrm{w}} \mathrm{N}_{\text {ré}}$, ógwnù = óg ${ }^{\mathrm{w}} \mathrm{u}$, ófnó = ófó. C'est pourquoi j'adopte pour l'essentiel dans ce travail, la transcription de l'API. Toutefois, $y$ remplace $j, c$ remplace $t$, et j remplace $d_{3}$; enfin 'b et $b$ sont préférés à $k p$ et $g b$ de l'orthographe officielle ikwere pour indiquer qu'il s'agit des consonnes non explosives bilabiales et non des labio-vélaires (pour plus de détail, voir Clements \& Osu 2002). Une voyelle qui est précédée d'une consonne nasale se nasalise automatiquement. Dans ce cas, nous préférons ne pas l'indiquer au moyen d'un tilde (voir Clements \& Osu 2005 ; Osu \& Clements 2009). Les tons sont indiqués comme suit : á = Haut ; à = Bas ; à = Montant ; $\hat{a}=$ Descendant $;{ }^{\downarrow}=$ Downstep (Haut Abaissé).

Les abréviations utilisées : $1 \mathrm{SG}=1^{\text {ère }}$ personne du singulier; $1 \mathrm{PL}=1^{\text {ère }}$ personne du pluriel ; $3 \mathrm{SGO}=$ $3^{\text {ème }}$ personne du singulier objet ; B.V. : base verbale ; EFF : effectif ; FUT $=$ futur ; IDF $=$ identification ; INACC $=$ inaccompli $;$ LOC $=$ localisateur $; \mathrm{NEG}=$ négation $;$ REDUP $=$ la copie dans une construction réduplicative $; \mathrm{REL}=$ relateur $; \mathrm{STAT}=$ statif. 
Les exemples en (2) et (3) illustrent l'emploi de cette unité verbo-nominale dans des énoncés. On s'aperçoit que l'élément àtâ peut apparaître dans d'autres constructions notamment àtâ rớtô 'mâcher le bâton à mâcher (ou frotte-dent)', àtâ ávỡ 'mâcher du poisson', àtâ órí 'mâchouiller (se dit d'une chèvre)'. Il en ressort que l'élément àtâ se caractérise par une diversité d'emploi que l'on ne peut pas simplement ramener à un cas de polysémie. En partant du postulat qu'il y a derrière cette diversité d'emploi un mode de fonctionnement invariant et spécifique, on peut mener une étude de cet élément dans des énoncés et contextes variés afin de dégager ce fonctionnement invariant et spécifique dans la langue. L'avantage d'une telle approche, c'est que l'utilisateur du dictionnaire aura à sa disposition une caractérisation susceptible de l'aider à mieux comprendre ce qui, dans l'unité àtâ, est réinvesti dans différentes constructions dans lesquelles elle apparaît.

Dans le dictionnaire, àtâ pourrait constituer une entrée à elle seule, c'est-à-dire sans $a h^{w}{ }^{w} h^{w}{ }^{\prime}$ 'souffrance', le complétant. ${ }^{6}$ Dans ce cas, les différentes combinaisons dans lesquelles elle apparaît seraient réunies sous cette entrée. Sauf qu'avec un tel choix, on prendrait le risque de court-circuiter les informations liées au complétant $a h^{w} \mho_{\text {'ó }}^{w}{ }^{w}$ 'souffrance', rớtô 'bâton à mâcher', $a ́ v \hat{\sigma}$ 'poisson' et '́rí 'objet invisible que les chèvres mâchouillent à longueur de journée'. On pourrait également créer des entrées différentes pour àtâ et $a^{\prime} h^{w}{ }^{\prime} h^{w} \dot{o}$. Dans ce cas, on aurait quelque difficulté à montrer comment les deux unités interagissent pour engendrer l'idée que l'on traduit ici par "souffrir ». La troisième option, à laquelle je souscris, consiste à les présenter ensemble comme une seule entrée. L'intérêt est que, ensemble ils forment une unité verbonominale que les locuteurs peuvent identifier facilement.

L'unité àtâ á $h^{w} ̛_{\text {ó }} h^{w}$ ' signifie littéralement 'mastiquer/mâcher l'asticot' dans la mesure où $a h^{w}{ }^{\prime} h^{w} \dot{u}$ désigne l'asticot. ${ }^{7}$ La question est de savoir quel peut être le lien entre «le fait de mastiquer l'asticot» et «souffrir ». En d'autres termes, l'utilisateur (surtout s'il est étranger à la culture ikwere) pourrait avoir quelque souci à appréhender l'unité verbale àtâ á $h^{w} \tilde{u}^{\prime} h^{w} \tilde{u}$. Car le lien de l'entrée lexicale ikwere avec son équivalent en français n'est pas du tout évident. Il me paraît donc nécessaire, dans ce genre de cas, de fournir quelques renseignements culturels supplémentaires afin d'aider l'utilisateur.

Cristinoï \& Nemo (2013: 126-132) insistent également sur l'importance de fournir des informations culturelles dans ce type de dictionnaire dans la mesure où celui-ci documente aussi bien une langue qu'une culture, et préserve un héritage linguistique et culturel qui pourrait disparaître s'il n'y avait pas ce matériel écrit.

1.1 Rappel succinct des travaux sur l'ikwere. Il existe en effet, très peu de travaux sur l'ikwere. Avant le début des années 1980, les seuls travaux connus sur cette langue étaient ceux de Kay Williamson (1970), réédité Williamson et al. (2010), un manuel scolaire destiné aux enseignants de l'école primaire, et ceux de S.A. Ekwulo (1970 réimprimé 1981 ; 1975), un recueil de données à partir des différents dialectes pour doter l'ikwere d'une orthographe suivi d'un recueil de proverbes ikwere traduits en anglais. Donwa-Ifode \& Ekwulo (1987) présentent une version plus récente de l'orthographe de l'ikwere. C'est à partir de ces données que quelques rudiments de phonétique et de vocabulaire ont pu être proposés. La création de la section de Linguistique et langues africaines à l'Université de Port Harcourt (Nigeria), elle-même fondée en 1975, a ouvert le

Voir plus loin pour une discussion sur la constitution de l'unité verbale en ikwere.

Il convient de préciser que l'idée de mastiquer est le résultat de l'interaction de àtâ et áh $h^{w}{ }^{\prime} h^{w}$ ó et ne serait en aucun cas attribué à àtâ seul. 
chemin à l'étude des langues de l'Etat des Rivières. Ainsi, une bibliographie a pu se constituer. Mais ce mouvement reste limité, car la grande majorité de ces travaux est constituée de mémoires rédigés en vue d'obtenir le diplôme de Bachelor of Arts (niveau Licence). Certes, ces travaux décrivent des éléments de la langue, mais cette description reste rudimentaire : ni la phonologie, ni le vocabulaire, ni la morphologie ne sont correctement documentés. Plus récemment, j'ai entrepris une étude dans la perspective de la construction du sens (domaine de l'énonciation) et dans le domaine de la phonologie en collaboration avec le regretté George Nick Clements. ${ }^{8}$

Enfin, depuis la soutenance de leurs thèses de doctorat, Alerechi (2007a) et Weje (2007) participent pleinement à la documentation de cette langue. Voir aussi Alerechi $(2007 \mathrm{~b}, 2008)$ et Weje (2003), Alerechi \& Weje (2013).

Il n'existe pas encore de dictionnaire pour l'ikwere. Cependant, dans Williamson (1970) réédité en 2010, cité plus haut, on trouve une liste de 245 mots répartis en 8 classes : numéraux, parties du corps, parenté, objets, nourriture, animaux, mots descriptifs, et verbes (présentés à l'impératif). Williamson signe ainsi la toute première tentative de répertorier les mots de cette langue.

Dans Ikwerre mbom, un manuel de 25 pages, Ekwulo (1970) lui emboîte le pas et propose une liste de 110 mots et expressions. Alors que Williamson part d'une liste de mots anglais pour les traduire en ikwere, Ekwulo adopte la démarche inverse. La liste de ce dernier contient de nombreux mots et expressions qui ne figurent pas dans la liste de Williamson, elle n'est pas répartie en classes de mots.

Dans Speaking Ikwere, un manuel d'initiation à la langue ikwere de 135 pages, Enyia (2008) propose une liste d'environ 1050 mots, la plus longue liste de mots ikwere publiée jusqu'à présent.

La principale difficulté avec ces manuels est qu'ils sont très difficilement accessibles. C'est surtout vrai pour les deux plus anciens (Williamson 1970 et Ekwulo 1970) qui sont, à ma connaissance, épuisés et donc introuvables sur le marché, y compris au Nigeria. En outre, la liste que propose Enyia (2008) est très peu exploitable du fait qu'elle contient beaucoup d'erreurs. Par exemple, l'auteur confond souvent les verbes et les adjectifs, présente des verbes tantôt sous leur forme conjuguée, tantôt sous leur forme non conjuguée sans pour autant justifier ses choix ; et il n'indique pas les tons, du moins selon les règles d'orthographe préconisées par Williamson (1970). Enfin, Enyia insère un point souscrit aux voyelles pour indiquer le trait [-ATR] alors que ces voyelles n'ont pas ce trait. La liste d'Ekwulo et celle d'Enyia ne sont pas établies selon un ordre spécifique.

\section{Le projet de dictionnaire}

Il s'agit dans cette section de préciser comment j'ai été amené à prendre la décision d'élaborer un dictionnaire trilingue et de présenter la structure de celui-ci. Je souligne également comment le corpus de départ a été élaboré.

2.1 La genèse du projet. S'agissant d'un linguiste qui décrit une langue peu documentée, on pourrait considérer le fait d'élaborer un dictionnaire bilingue comme faisant partie des nombreuses tâches qui lui incombent et donc comme un exercice qui va de soi. Mais dans le cas présent, la décision d'élaborer un dictionnaire pour l'ikwere s'est imposée à moi lors d'un exercice dans un

8 Quelques références sont citées dans la bibliographie ci-dessous. 
cours de français il y a environ trente ans. ${ }^{9}$ L'exercice consistait à traduire un chant dans nos diverses langues maternelles. J'ai éprouvé d'énormes difficultés à le faire. D'ailleurs, contrairement à mes camarades, je n'avais pas de dictionnaire bilingue à ma disposition. Les circonstances qui m'ont amené à prendre cette décision sont donc celles de la traduction. S'ajoute à cela le fait que très peu de personnes écrivent l'ikwere. Pour ces raisons, un dictionnaire monolingue me paraissait non seulement inapproprié mais aussi d'un intérêt très limité à court et à moyen terme. D'un autre côté, un dictionnaire bilingue ikwere-français peut paraître adéquat, mais comme le Nigeria est une ancienne colonie britannique, un locuteur ikwere parle et lit plutôt l'anglais. Enfin, un dictionnaire bilingue ikwere-anglais ne pouvait pas non plus répondre à mes propres besoins en France. Devant une telle situation, le dictionnaire trilingue ${ }^{10}$ ikwere-anglaisfrançais $^{11}$ dont il est question ici peut paraître trop ambitieux mais il est plus approprié car il cherche à offrir aux utilisateurs des communautés linguistiques concernées, les moyens de communiquer avec plus de facilité. Ce dictionnaire s'adresse également aux spécialistes de langues (africaines et autres) ainsi qu'aux étudiants en linguistique africaine.

Il est donc envisagé que ce dictionnaire reflète les divers aspects de la vie et de la culture ikwere, allant des activités de la pêche, de la culture agricole, de la chasse à la vie quotidienne (y compris la cuisine, la nourriture, les recettes, la danse des masques et d'autres types de danses, le repos, la naissance, le mariage, la mort, les croyances, le marché, les soins et médecines traditionnels) en passant par les contes, les devinettes, les dictons, les proverbes, les récits de la vie quotidienne. A terme, ce dictionnaire contiendra des items lexicaux et grammaticaux, mais aussi, des noms de personne, des toponymes, des ethnonymes, des items issus de l'(ethno)botanique, pour ne citer que ceux-là.

On pourrait penser que l'objectif assigné à ce dictionnaire trilingue dépasse celui qu'on assigne à ce type de dictionnaire. Mais, là encore, Cristinoï \& Nemo (2013 : 132) préconisent de dépasser le cadre strict d'un dictionnaire bilingue -allusion à une liste d'unités avec leurs équivalents dans la langue d'arrivée- pour se placer dans une perspective encyclopédique.

Le corpus de base a été recueilli avec le questionnaire d'inventaire linguistique (questionnaire 5 : mots et phrases) adapté aux besoins de ce projet. C'est un questionnaire de 221 unités, élaboré en 1981 par la SELAF (Société d'Etudes Linguistiques et Anthropologiques de France), d'après l'ouvrage en 3 volumes intitulé Enquête et Description des Langues à Tradition Orale, vol. II, pour les langues sans classes nominales. Ce premier corpus a été étoffé au fil des ans grâce à des séances de discussions et d'interrogations-réponses initiées en interaction avec les locuteurs natifs lors de mes divers séjours au Nigeria.

9 A l'époque, j'apprenais le français au CLAB (Centre de Linguistique Appliquée de Besançon, France), loin de toute préoccupation de la documentation de l'ikwere.

10 On pourrait s'interroger sur le concept de dictionnaire plurilingue et sur le rapport et le degré d'adéquation entre ce concept et la pratique, c'est-à-dire l'élaboration d'un dictionnaire tel que celui que je présente ici. Pour une discussion à ce sujet, le lecteur pourra se reporter à Béjoint \& Thoiron (1996), en particulier à la contribution de Marello.

11 Voir aussi Bouquiaux (2002: 17-24), qui s'exprime au sujet de son dictionnaire trilingue birom-françaisanglais. Il écrit (p.18) : «Ce n'est que dans les années quatre-vingt-dix que je me résolus à utiliser le reste de ma documentation pour en tirer un dictionnaire trilingue (birom-français-anglais), avec la perspective de pallier les insuffisances que j'avais relevées dans les ouvrages que j'avais utilisés jusque-là et qui m'avaient laissé largement insatisfait. Ils ne répondaient en fait pour la plupart qu'aux vœux de linguistes ne s'intéressant qu'à la langue, à des fins comparatives. Mon ambition était beaucoup plus vaste... ». Le birom est une langue Niger-Congo parlée dans l'Etat du Plateau dans le centre du Nigeria. 
Comme il s'agit avant tout d'un dictionnaire de langue, les entrées sont illustrées d'énoncés authentiques (et non de phrases hors contexte) car, comme l'écrit Grundy (1996: 132), «l'utilisateur est en droit d'attendre de son dictionnaire qu'il répertorie les usages réels et non ceux que le lexicographe a imaginés. Quelle que soit l'ingéniosité avec laquelle on peut prévoir les besoins de cet utilisateur, il est difficile d'envisager que ces besoins, dans le cadre de la compréhension de la langue source ou celui de la communication en langue cible, puissent être fondés sur autre chose que des exemples d'utilisation du langage en situation réelle ».

Ces énoncés authentiques sont d'abord soumis à un découpage morphologique, ce qui permet de restituer des formes non perceptibles en surface, puis à une traduction mot à mot dans une langue d'arrivée, l'anglais en général, avant d'être traduits aussi bien en anglais qu'en français.

2.2 La structure du dictionnaire. Dès le départ, ce projet de dictionnaire est réalisé dans une base de données créée à l'aide de Toolbox (Summer Institute of Linguistics). Il comprend actuellement 2360 entrées et il est prévu un nombre assez important de champs pour une entrée afin de donner le maximum d'information sur la langue et sur la culture ikwere sans pour autant viser à renseigner tous les champs pour toutes les entrées. Voici, en aperçu, la nature des champs rattachés à une entrée.

Une entrée s'ouvre sur un champ intitulé « lex » pour lexème. Le mot « lexème » n'a pas ici un sens technique. Il désigne simplement le champ qui contient un mot (une entrée, une vedette ou une unité lexicale) de la langue ikwere, présenté à l'aide d'une transcription phonétique. Le champ « lexème » est suivi du champ «phm » qui propose une transcription phonologique de l'unité en question. Ensuite vient le champ « $\mathrm{mb} »$ dans lequel est indiquée la frontière de morphème, puis le champ « ortho » pour l'orthographe officielle ikwere de l'unité en question. De cette façon, l'unité pourra être facilement identifiée aussi bien par les linguistes et autres utilisateurs initiés à la transcription phonétique que par les locuteurs ikwere.

Il est également prévu un champ « $d v$ » pour les variations dialectales et un champ «morph» pour la structure morphologique de l'entrée lorsque celle-ci est une unité composée. Le champ « gleng » est prévu pour la traduction en anglais tandis que «gfr » donne la traduction en français. Il y a également « engtrans et frtrans » deux champs pour des exemples illustratifs dans ces deux langues, puis un champ «son » pour enregistrer les entrées produites par des locuteurs natifs et un champ « pix » lorsqu'on a des images, photos ou vidéos qui illustrent l'entrée.

Deux champs «ng1 » et «ng » sont prévus pour des notes et commentaires assez élaborés qui peuvent être d'ordre culturel ou linguistique (autre que phonologique, car il existe un champ pour des commentaires et notes sur des phénomènes phonologiques). Ainsi, par exemple, les commentaires sur les noms de personne sont insérés dans ces deux derniers champs.

Il y a un champ « tp » pour indiquer le schème tonal de l'entrée. Mais dans la mesure où il est extrêmement difficile de concevoir les mots hors énoncés, un tel schème tonal ne peut être qu'indicatif. En outre, un champ «ps » est proposé pour indiquer la partie du discours à laquelle l'unité peut être a priori affectée selon le classement traditionnel.

Les unités de l'ikwere se répartissent en verbes, noms et qualificatifs. ${ }^{12}$ Morphologiquement, le verbe est, dans la majorité des cas, c'est-à-dire si on excepte les verbes dits de délocalisation (àbyâ

12 Quel dilemme que celui d'éviter pour la langue d'étude de calquer sur les métalangues et/ou sur les catégories préétablies en même temps que l'on cherche à identifier les classes de mots (ou parties du discours) pour cette même langue selon une tradition qui remonte à l'Antiquité. Bien malin qui y arrive sans trébucher. Je ne prétends pas y arriver dans mes travaux, j'essaie de m'appuyer le plus possible sur 
'venir', àlâ 'rentrer (centrifuge)', àyâ 'rentrer (centripète)', èh ${ }^{w} \hat{e}$ 'passer'), composé de deux unités (base + complétant/complément). Ce complétant est en général un nom ou un qualificatif, y compris les noms qui assument la fonction de qualificatif. ${ }^{13}$ Alors que le complétant reste invariable, la base peut subir toute une série de flexions et porter les marques d'aspect, de temps, de modalité et de personne. ${ }^{14}$ Sémantiquement, le verbe représente, au sein de l'énoncé, le procès (qu'il s'agisse d'action, d'état ou d'événement) dans une relation sujet-procès.

Le nom ne varie pas morphologiquement, sauf les noms dérivés des verbes (ex. èrí wírí 'manger' mais òrí wirí 'mangeur' (glouton)). Le nom peut occuper la position «sujet» et « complément » du verbe. Il peut être suivi d'une marque de personne pour indiquer une relation d'appartenance ou de possession (غ̀rèkpàm 'mon sac', órò wórí '(la) maison de Wori'). Le nom peut soit qualifier un autre nom (ólú śrj̀ 'le dessus d'une maison'), soit être qualifié par un autre nom ('́rj̀ ólú 'maison hauteur' c'est-à-dire maison à étage). Il peut comporter un préfixe relativement identifiable, trace d'un système de classes nominales désuet, comme dans $\dot{n}$-dá 'père', ń-né 'mère',

La classe des qualificatifs regroupe les termes suivants : ńdíndi 'de nature courte', ógólógó 'de nature longue', ábádámbá 'de nature plate' ${ }^{15}$ mais aussi ráhí ràhì ráhí 'zigzag', kítí kítí 'picotement', bìrí bìrí 'd'un goût sucré', réh á réh $h^{w} \grave{a}$ 'blanchâtre'. Ce qui les caractérise, c'est qu'ils complètent le verbe. Si on peut considérer certains d'entre eux comme idéophones (du fait de leur structure réduplicative et de l'impossibilité de les affecter à la classe des verbes ou des noms) p.ex. bìrí bìrí 'd'un goût sucré', on a du mal à faire de même pour d'autres, p.ex. ráhí ràhì ráhí 'zigzag' et ógólógó 'de nature longue'.

Certaines unités sont toutefois des mots de liaison (ou relateurs, connecteurs). C'est le cas de l'unité $n u$. Enfin, on a un champ «mchar» pour la caractérisation métalinguistique de l'unité. L'originalité de ce champ est qu'il propose une définition qui s'appuie sur une étude systématique de l'unité en question ( $c f$. discussion supra). De ce fait, cette définition peut parfois s'avérer très technique.

Prenons pour illustration l'unité $n \sigma$. D'après Osu (2003a), ce morphème correspond à cette catégorie d'unités traditionnellement considérées comme 'mots de liaison' ou encore 'mots fonctionnels'. Elle peut ainsi relier des éléments qui sont de la même catégorie grammaticale (p.ex. nom + nom) ou de catégories différentes (p.ex. verbe + nom). Elle peut apparaitre en début d'un énoncé ou en position finale absolue. Ainsi, on se demande s'il s'agit d'un seul morphème avec une diversité d'emplois ou de plusieurs morphèmes homonymes. Une étude systématique de cette unité a montré que derrière le foisonnement d'emplois qui la caractérise, il y a une propriété

les données, seule issue à ce dilemme. On se souvient dans la section du Sophiste (p.327) que Platon consacre à l'analyse du discours, du jugement et de la représentation imaginative, du personnage L'Etranger qui, s'adressant à Théétète, signale qu'il existe deux genres, deux sortes de moyens par lesquels on parvient à signifier quelque chose, à exprimer la réalité existante par l'intermédiaire de la voix ; ce sont pour l'un les noms et pour l'autre, les verbes. L'Etranger établit ainsi une nette distinction entre le verbe, défini comme ce qui rend manifestes les actions, et le nom, qui s'applique au sujet accomplissant ladite action. Pour une discussion très accessible des parties discours en français, le lecteur pourra se reporter à, entre autres, Choi-Jonin \& Delhay (1998) et Lemaréchal, A. (1989).

13 Par exemple, míní wíri 'eau nourriture' (sauce); ńw ָ̇́ rùkà 'enfant mâle' (garçon) versus ńwó rìyà 'enfant femelle' (fille).

14 Osu (à paraître) montre que l'ikwere marque l'accord de la première personne du singulier sous certaines conditions particulières.

15 Voir Emenanjo (1978 : 47) pour une catégorie similaire en igbo. 
invariante. En effet, ce morphème implique la redélimitation d'un premier terme qui est mis en relation avec un deuxième terme. A ce titre, il fournit le repère nécessaire pour construire une relation de repérage. Par souci de précision, cette propriété invariante est reformulée techniquement de la manière suivante : «étant donné $\mathrm{E}_{1}$ (un premier terme, c'est-à-dire une situation, la valeur d'un chiffre, un instant, une relation prédicative, etc.), $n u$ introduit $\mathrm{E}_{2}$ (un deuxième terme) et le fonde comme le terme à prendre en compte de façon à redélimiter $\mathrm{E}_{1}$ ». Les diverses traductions (sur, et, que, par, à, pour, mais) que je propose pour cette unité dans des exemples sont ainsi articulées à cette caractérisation. Voir en annexe l'entrée $n u$ et sa caractérisation métalinguistique.

Rey-Debove et Rey (2007), cité par Dotoli (2008 : 39) notent : « un véritable dictionnaire de langue va beaucoup plus loin (que les définitions des mots) en décrivant toutes les manifestations du sens et sa circulation dans le lexique ».

\section{Quelques aspects de la culture ikwere dans le projet de dictionnaire}

Ce projet de dictionnaire se veut donc le reflet des aspects de la culture ikwere. A travers certaines entrées, un aspect de cette culture est présenté dans le champ «ng1» pour notes and commentaires généraux.

3.1 Noms de personne. Prenons le cas des noms de personne. ${ }^{16}$ La question est de savoir quel traitement en est fait dans ce projet de dictionnaire et comment mes travaux de description en linguistique et en ethnolinguistique me permettent de traiter de ce type de données. Avant de revenir sur ce point, je voudrais tout d'abord donner un aperçu du nom en ikwere.

Le nom d'un individu, d'un enfant en l'occurrence, est en fait un énoncé, toujours tronqué dans l'usage. Le nom d'un individu est donc souvent l'extrait d'un énoncé. Il ne semble pas y avoir de règles spécifiques pour cette troncation. Il peut s'agir d'une aphérèse, d'une apocope, ou des deux. Le résultat d'un énoncé ainsi tronqué peut être une unité monosyllabique (p.ex. $c \hat{\imath}$ 'ange-gardien, Dieu'), dissyllabique (p.ex. àvộ 'dos') ou plurisyllabique (p.ex. ǹyékácî̀ 'qui est plus grand que Dieu'). Il peut créer une illusion dans la mesure où deux énoncés raccourcis en deux noms portés par deux personnes différentes peuvent se ressembler, donnant l'impression que l'on a deux fois le même nom. Une telle illusion est vite dissipée lorsqu'on considère les deux énoncés en entiers. Par exemple le nom bádợ peut être la forme abrégée de bádờmênwó 'les humains ne créent pas les enfants' ou bádòmènònòmà '(il y a) quelqu'un (qui a eu la force de) le faire pour moi'.

Le nom est, en règle générale, créé ${ }^{17}$ et attribué à l'enfant par la personne qui assume les responsabilités de père à son égard. ${ }^{18} \mathrm{~A}$ défaut, c'est au grand-père paternel, s'il est encore en vie, que revient cette responsabilité. Sinon, cela peut être la mère ou la grand-mère paternelle. En

16 Pour une importante discussion des phénomènes liés aux noms, voir Akin (1999).

17 Par «créé » je veux signifier que le nom n'est pas nécessairement choisi dans une liste de noms préexistante.

18 Le père d'un enfant n'est pas nécessairement son père biologique ou géniteur. Ainsi, par exemple, si une femme non mariée a un enfant, la responsabilité de parents revient aux parents de cette femme. De même, lorsqu'une femme divorce et se remarie, elle peut amener ses enfants avec elle pour s'installer avec son nouveau mari. Ces enfants deviennent dans la pratique ceux du nouveau mari. Mais cela ne les empêchera pas de garder contact avec leur père biologique et de retourner un jour chez lui s'ils le souhaitent. Les choses sont donc plus complexes qu'elles n'y paraissent. 
parallèle, un autre adulte, pour quelque raison que ce soit, dès lors qu'il est de la même famille paternelle élargie que l'enfant, peut lui donner un nom.

Le nom d'usage, celui par lequel l'individu sera connu et identifié, lui est donné le jour où son cordon ombilical tombe et est enterré sous une plante dite économique, comme par exemple isisí ćkìnìmá (arbre pour la banane plantain), isisí ák $k^{w} u ̛$ mèkéy (le cocotier ou le palmier pour le vin de palme). Cette plante appartient désormais à l'enfant et constitue pour lui un cadeau-épargne ou un investissement de la part de ses parents. Il aura ainsi de quoi démarrer la vie lorsqu'il deviendra adulte. ${ }^{19}$ Il pourra vendre les bananes du bananier et le vin qui est extrait du palmier.

Le nom d'un individu peut simplement refléter sa position dans l'ordre des naissances au sein de la famille dont il est issu (du côté du père, du côté de la mère ou des deux côtés). Par convention, le premier des garçons (l'aîné) s'appelle ś ’ụárá (mais parfois óké ou wóké dont on trouve des formes proches dans des dialectes igbo). Le cadet s'appelle j̀ló ou wòló, celui qui vient après le cadet s'appelle j̀lú ḿmántì ou wòló mmmántì (ḿmántì désigne la petitesse), et le benjamin s'appelle j̀lú ḿmántì kírí kírí (kírí kírí est un terme idéophonique qui tend à renforcer l'idée de petitesse).

La première des filles (l'aînée) s'appelle àdá ou wàdă ; la cadette, òlú ou wòlú ; la suivante, òlú ḿmántì ; et la benjamine òlú ḿmántì kírí kírí.

On notera la différence introduite par les voyelles entre le fils cadet (mâle) et la fille cadette (femelle). Pour le fils cadet, la voyelle est postérieure, arrondie et mi-basse tandis que pour la fille cadette, la voyelle est également postérieure, arrondie mais mi-haute. De plus, pour le fils cadet, la voyelle est caractérisée par le trait [-ATR] tandis que pour la fille cadette, nous avons une voyelle avec le trait [+ATR]. On peut donc dire que dans ce cas précis, l'ikwere se sert d'un moyen phonologique pour distinguer entre mâle et femelle.

Ajoutons qu'un individu peut cumuler les deux types de nom, c'est-à-dire, un nom lié à l'ordre de sa naissance et un nom ou plusieurs créés en fonction des circonstances qui entourent sa naissance. $^{20}$

Le nom d'un individu peut renvoyer à un élément de la nature. C'est le cas de íbíné donné à quelqu'un pour indiquer qu'il est un parangon de beauté. Le terme íbiné désigne un type de fruit qui ressemble à un raisin ; quand il est mûr, sa peau verte s'ouvre et se replie vers la tige laissant apparaître une chair blanche. Son jus est très sucré.

Mais chez les Ikwere, le nom d'un individu, dans la grande majorité des cas, représente l'expérience de vie du père ou de la personne qui le crée et le lui attribue. Ainsi si un père a le sentiment que pour une raison quelconque, lui et sa famille font l'objet de dérision ou de moquerie de la part de leurs voisins, il peut choisir de nommer son enfant ìbùcînékè (raccourci : ìbòcî) 'es-tu Dieu'? (Sinon pourquoi est-ce que vous vous moquez de nous)?

Le nom peut également être la trace d'un événement spécifique lié à la naissance de l'individu qui le porte. Ainsi, un garçon a été nommé j̀kśbúlêm mé (raccourci : jksóbúlé) 'il m’a trop fait

19 Du fait de l'urbanisation à laquelle est confronté le peuple ikwere, cette pratique, comme beaucoup d'autres pratiques de la culture ikwere, est en train de tomber en désuétude. En effet, il faut plus d'espace aujourd'hui qu'hier pour construire une seule maison. En plus, les Ikwere connaissent une explosion démographique. Si on ajoute à tout cela le besoin de vivre dans un environnement plutôt débarrassé des brousses, on comprend que les plantes économiques aient de moins en moins de place au sein de la famille ikwere.

20 Il faut ajouter aussi un troisième type de nom, le nom de baptême pour les familles chrétiennes; c'est le nom avec lequel l'individu s'inscrit à l'école. A ma connaissance, il s'agit dans tous les cas d'un nom d'origine européenne. 
défaut/manqué' pour indiquer que malgré son désir d'avoir un garçon, le père n'a eu droit jusquelà qu'à des filles.

En tout cas, le nom de personne peut exprimer les vœux, les souhaits, les désirs, les remerciements, la revanche des parents ou indiquer tout simplement ce que ceux-ci attendent de la vie.

Il ressort que dans la culture ikwere, le nom de personne n'est pas une unité toute faite ou choisi au hasard, il n'est pas non plus choisi par rapport à sa sonorité. On notera en passant qu'il n'y a pas de nom spécifiquement pour les femmes et d'autres pour les hommes, si l'on excepte les noms liés à l'ordre de naissance.

Cette conception du nom de personne n'est cependant pas exclusive de la culture ikwere. S'agissant des noms propres en amharique (Ethiopie), Negga (2008) écrit : « [...], le nom reflète généralement les circonstances politiques, socio-économiques ou familiales du moment ; ou bien l'état d'âme de celui qui donne le nom ; ou encore, l'espoir que l'enfant incarne ; dans tous les cas, le nom traduit le message que l'on tient à faire passer. »

Tiérou (1977 : 14) s’interroge sur ce phénomène. Il écrit : "Pourquoi chaque nom africain revêt-il une signification concrète ou abstraite? » D'ailleurs au pays des Ouehon (c'est le vrai nom du peuple Guéré en Côte d'Ivoire), le nom de personne serait-il d'utilité didactique ? Pour une lecture complémentaire sur les noms de personne, le lecteur pourra consulter également Asante (1991), Leguy \& Lebarbier (2006) et Stewart (1996).

3.2 Noms de personne dans le projet de dictionnaire. Prenons le cas de ńhnệánòtnú dans l'orthographe officielle ikwere (l'équivalent Alphabet Phonétique International : ńh ॄิánòtú) qui signifie 'ce que nous cherchons'. ${ }^{21}$ Ce nom contient trois unités :

ńhnệ [ńĥ̃̂] 'chose'

á (forme réduite de áyì) 'nous'

nòtnú [nòtú] (unité verbale composée du préfixe de l'inaccompli $n$ - et la forme non fléchie du verbe òtnú) : l'idée de chercher.

Ce nom (transcrit en API cette fois) est extrait de l'énoncé complet suivant : ${ }^{22}$

(4)

\begin{tabular}{|c|c|c|c|c|}
\hline ńhęָ̧án & ớńw: & & & \\
\hline 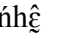 & á & n-òtứ & bú & ńwă \\
\hline chose & $1 \mathrm{PL}$ & INACC-B.V.chercher & être.IDF & enfant \\
\hline
\end{tabular}

Nous avons là une structure focalisante en ikwere, signalée par l'élément $h$ h $h \hat{\varepsilon}$ à l'initiale et le verbe d'identification bó qui introduit ńwó. L'élément $n h \hat{z} \hat{\varepsilon}$ indique que le sujet $<$ nous $>$ veut quelque chose qui n'est pas précisé. Cela signale une relation prédicative préconstruite qu'on peut noter

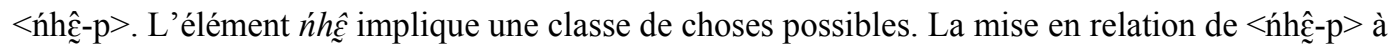
ńwź par le verbe bú permet de sélectionner ńwó dans la classe et de le désigner comme ce que le sujet $<$ nous $>$ veut. L'ensemble de la relation de focalisation peut être noté : $<_{1}<{ }_{0}$ ńh $\hat{\varepsilon}-\mathrm{p}_{0}>$ bú

21 Ceci est le nom ikwere du présent auteur.

$22 \quad 1 \mathrm{SG}=1^{\text {ère }}$ personne au singulier ; $1 \mathrm{PL}=1^{\text {ère }}$ personne au pluriel $; 3 \mathrm{SGO}=3^{\text {ème }}$ personne au singulier objet ; FUT $=$ futur ; IDF $=$ identification ; INACC $=$ inaccompli ; LOC $=$ localisateur ; NEG = négation ; REDUP $=$ la copie dans une construction réduplicative $; \mathrm{REL}=$ relateur $; \mathrm{SPEC}=$ spécifieur $; \mathrm{STAT}=$ statif. 
ńw ${ }_{2}>$. En effet, bó établit une relation d'identification entre la classe des possibles et ńwó, de sorte que la sélection de ńwó aboutit à l'élimination des autres choix possibles : c'est un enfant que nous voulons et pas autre chose. Pour une analyse de la focalisation en ikwere, voir (Osu 2000a). Ces remarques d'ordre linguistique sont insérées dans le champ «ng ».

Une des raisons qui peut amener les parents à donner ce nom à leur enfant est la suivante : la mère a fait plusieurs fausses couches et ils ont perdu un de leurs enfants. La mère étant à nouveau enceinte, les deux parents ainsi que leur entourage craignent une catastrophe, ils craignent que l'enfant ne meure à la naissance ou que la mère ne meure lors de l'accouchement. Cet énoncé est donc une façon d'exprimer leur joie, mais aussi une façon de se rassurer. On indique à qui veut l'entendre que les parents veulent bel et bien des enfants (comme celui qu'ils viennent d'avoir) et pas les malheurs. Cet énoncé peut donc s'interpréter comme ceci : «c'est bien des enfants que nous voulons et pas des malheurs ». Ce type d'information relève spécifiquement de la culture et est inséré dans le champ «ng1».

Le nom ńhêánòtú a pour variante ihéánàcó. Il s'agit là de la forme correspondante igbo mais qui peut tout à fait s'employer chez les Ikwere. Cela peut s'expliquer par le fait que les deux langues sont apparentées d'une part, mais aussi par le contact permanent entre les deux langues, d'autre part. En effet, le peuple ikwere et le peuple igbo vivent dans des régions géographiquement proches l'une de l'autre, partageant des frontières; de plus, les activités commerciales les amènent à se rencontrer fréquemment. Ils ont également eu pendant assez longtemps des rapports politiques dans lesquels le peuple igbo, et donc sa langue, occupait une position beaucoup plus importante que le peuple ikwere puisque la capitale régionale se trouvait en terre igbo. Il est hautement probable, dans ces circonstances, que les deux langues se sont influencées mutuellement, certainement à des degrés divers.

Cette information est donnée dans le champ « comvar » créé pour les informations qui portent sur les formes variantes.

Prenons un deuxième exemple : ǹyê $k^{w} e^{23}$ Il est extrait de l'énoncé h̀yễ $k^{w} e ́$ nò kè á qui se présente comme ceci :

$$
\begin{aligned}
& \text { ǹyê } \mathrm{k}^{\mathrm{w}} \mathrm{e} \text { nò kè á } \\
& \text { ǹyễ } \mathrm{k}^{\mathrm{w} e ́ ~ n o ̀ ~ k e ̀ ~ a ́ ~} \\
& \text { qui B.v.accepter REL SPEC 3SGO }
\end{aligned}
$$

Qui accepte une chose pareille dans son propre cas, ou quand cela lui arrive?

A partir de ce nom, on peut engendrer les énoncés suivants : ǹdâ ńyé $k^{w} e ́$ nò kè á 'Où est la personne qui accepte une chose pareille quand cela lui arrive ?' ; ou j̀ẑ́ ńyé $k^{w} e$ nò kè á 'Il n'existe pas une personne qui accepte une chose pareille lorsque cela lui arrive'; ou encore h̀yê zè̀ $k^{w} e ́$ nò kè á 'Qui voudra accepter quand cela le concernera ?'. Les trois énoncés sont considérés comme trois différentes manières de contextualiser ce nom. Par conséquent, ils peuvent tous s'adresser à celui qui porte ce nom. Disons qu'ils veulent tous dire la même chose. A chaque locuteur d'employer celui qu'il veut. Mais si le sens construit des trois énoncés n'est que très légèrement différent, le mécanisme de la construction de ce sens, lui, diffère d'un cas à un autre.

En effet, le point commun des trois est qu'il s'agit d'un nom porté par un enfant dont les parents, pour le sauver lorsqu'il était malade au point qu'on attendait sa mort, ont dû contourner

23 Ce nom peut encore être abrégé en é $k^{w} e ́$. N'étant pas en mesure, pour l'instant, d'expliquer l'apparition de la voyelle é à ton haut à l'initiale de l'unité, l'on se contentera ici de relever ce phénomène. 
les règles établies de fonctionnement de la société. En fait, sa grand-mère paternelle devait faire un sacrifice à ses ancêtres. Or, le seul membre de sa famille d'origine habilité à présenter ce sacrifice aux ancêtres a refusé de le faire. Ils ont donc eu recours à un autre membre qui, bien que n'étant pas habilité, a accepté de présenter le sacrifice aux ancêtres. Ils ont transgressé une règle et s'exposaient à des critiques. C'est donc pour répondre à ceux qui voudraient critiquer leur démarche, au présent comme à l'avenir, que les parents ont créé ce nom sous forme de question rhétorique, pour signifier que personne n'accepterait de voir son enfant mourir, et qu'on fait tout ce qu'on peut pour le sauver. Ce passage est inséré dans le champ «ng ».

Avec l'élément h̀yê qui a le schème tonal Bas-Descendant, le locuteur introduit une classe de personnes et leur prête la propriété q <être susceptible d'agir autrement>. Or, ǹyê introduit également de l'indétermination car le locuteur tend à dire qu'il a envisagé, a parcouru une à une les personnes appartenant à cette classe abstraite introduite, pour constater à l'issue de son parcours qu'aucun membre ne valide finalement la propriété q <être susceptible d'agir autrement>. Le spécifieur kè souligne une relation nécessaire entre la personne introduite par $a ́$ (3SGO) et le type de situation en jeu : sa situation à soi. Quant au relateur $n \sigma$, il assume dans cet exemple une fonction de type prépositionnel, et on peut le traduire par 'dans', ce qui donne : 'dans sa situation à soi'.

A travers ǹdâ dans ìdâ nyé $k^{w} e ́$ nò kè á 'Où est la personne qui accepte une chose pareille quand cela lui arrive?', le locuteur tend à dire qu'il n'est pas en mesure de situer ou encore d'identifier une personne dont il pourrait dire qu'elle accepterait la situation en question. On peut dire la même chose de j̀z dans jzế nyyé $k^{w} e ́$ nò kè á 'Il n'existe pas une personne qui accepte une chose pareille lorsque cela lui arrive' puisque l'élément j̀ẑ́ indique, là encore, la non-existence d'un individu dont le locuteur pourrait dire qu'il accepterait la situation en question.

Quant au préfixe verbal $z$ - dans ǹyê zè $k^{w} e ́$ nò kè á, 'Qui acceptera/voudra accepter (une telle situation) quand cela le concernera ?', il permet au locuteur d'indiquer que la relation x-p (x représente $<$ une personne $>$ tandis que $\mathrm{p}$ représente $<$ accepter que son enfant meure $>$ ) n'est pas inscrite dans le temps. Ce faisant, le locuteur signifie qu'on ne peut pas poser une telle relation comme étant de l'ordre de l'envisageable de sorte à évaluer le degré de possibilité de son ancrage dans le temps. ${ }^{24}$ Cette information est linguistique et est insérée dans le champ «ng1».

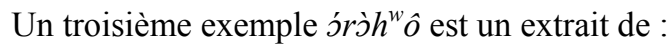

$$
\begin{aligned}
& \text { órò } \mathrm{h}^{\mathrm{w}} \mathrm{o} \text { ò } \mathrm{h}^{\mathrm{w}} \mathrm{u} \\
& \text { órò } \quad h^{w} \text { ú-ò } \quad \text { ò }^{\mathrm{w}} \mathrm{ù} \\
& \text { maison disparaître-NEG REDUP } \\
& \text { Une lignée (une famille) qui ne disparaîtra jamais. }
\end{aligned}
$$

L'arrivée d'un garçon, dans une famille qui n'a eu jusque-là que des filles, est source de soulagement. En effet, les filles sont censées quitter leur famille d'origine pour s'installer dans la famille de leur mari, alors que les garçons, eux, restent ; ils sont donc les gages de la continuité de la famille. Cette information est dans le champ «ng " comme illustré par l'entrée śrj̀ $h^{w} \hat{o}$ en annexe.

On note la négation signalée par le ton descendant sur la voyelle finale de la base verbale. On note également l'emploi réduplicatif du verbe «disparaître ». Cette structure permet au locuteur d'asserter contre autrui, réel ou imaginaire, que la famille, la sienne est appelée à se perpétuer. La

24 Voir Osu (2003b) pour l'analyse de $z-$. 
réduplication en ikwere consiste à poser une unité linguistique, appelée la base (notée $\mathrm{U}_{1}$ ), et dans le même mouvement à poser une autre unité, appelée la copie (notée $U_{2}$ ) qui dans sa forme ressemble soit totalement soit partiellement à la première, de telle sorte que la copie soit étroitement liée à ou dépende entièrement de la base $\left(\mathrm{U}_{1}-\mathrm{U}_{2}\right)$. La base peut apparaître seule ou en combinaison avec une autre unité à la place de la copie. Par conséquent la forme $\mathrm{U}_{1}-\mathrm{Y}$ est tout à fait possible. En revanche, la copie n'apparaît jamais seule sans la base ni avec une base qui lui est dissemblable. Par conséquent, la forme $\mathrm{Y}-\mathrm{U}_{2}$ ne peut pas constituer une réduplication. Le ton descendant sur la voyelle finale de la base verbale signale la négation. ${ }^{25}$ L'ensemble de cette construction permet au locuteur d'affirmer que sa famille est appelée à se perpétuer.

En effet, à travers la première mention de « disparaître », le locuteur introduit une occurrence du domaine notionnel représenté par « disparaître ». Or cette occurrence est de caractère instable. La copie permet d'introduire l'occurrence représentative de «disparaître ». La réduplication, considérée comme l'interaction de la base et de la copie, permet de rapporter la première occurrence à l'occurrence représentative du domaine « disparaître ». Le domaine se présente cette fois comme stabilisé, et on peut l'interpréter comme ceci : si on considère ce qu'on appelle « disparaître », l'arrivée d'un garçon fait que la famille pourra l'éviter.

Par ailleurs, le nom ikwere peut être associé au nom du père ou de la mère de celui qui le porte lorsque l'on a besoin de donner plus de moyen pour identifier l'individu qui le porte. Ainsi, on peut dire indistinctement ék $k^{w} e ́$ ńwó wóké ojiébè 'Ekwe le fils de Woke Ojiegbe' (Woke Ojiegbe est le père) ou é $k^{w} e ́$ ńwź wógà 'Ekwe le fils de Woga' (Woga est la mère).

Voici, pour fixer les idées, quelques autres noms dans l'orthographe officielle de l'ikwere avec l'équivalent en API dans la deuxième colonne, puis la traduction en français et en anglais.

\begin{tabular}{|c|c|c|c|}
\hline Aleruchi & [álérúcî] & $\begin{array}{l}\text { Attendons après le } \\
\text { Seigneur }\end{array}$ & Let's look unto the Lord \\
\hline Badnụkenwọ & [bádờkêńwõ] & $\begin{array}{l}\text { Personne ne crée les } \\
\text { enfants }\end{array}$ & No human creates children \\
\hline Bụdnuka & [búdờkâ] & La vie vaut plus que tout & $\begin{array}{l}\text { Life is worth more than everything } \\
\text { else }\end{array}$ \\
\hline Cherukei & [cèrúkěy] & Occupe-toi de tes affaires & Mind your own business \\
\hline Zeruọwa & [zèrúòwà] & Méfie-toi des gens & Beware of people you meet \\
\hline
\end{tabular}

3.3 Toponymes. Chez les Ikwere, èzí n-órò 'la maisonnée', c'est-à-dire un groupement d'individus qui habitent a priori sous le même toit, est le plus petit groupement nommable. Et un groupement de maisonnées ayant le même grand-père se nomme d'après le grand-père, précédé de rómò '(les) enfants'. Ainsi, la maisonnée $\mathrm{X}$, la maisonnée $\mathrm{Y}$ et la maisonnée $\mathrm{Z}$ ayant le même grand-père $\mathrm{A}$, s'appelle rómò-A '(les) enfants de A'; la maisonnée X', la maisonnée Y' et la maisonnée Z' ayant le même grand-père $\mathrm{B}$ s'appelle rómò-B '(les) enfants de B'. Dans la mesure où $\mathrm{A}$ et $\mathrm{B}$ sont, en principe, issus du même père $\mathrm{C}$, on peut les appeler rómò̀-C '(les) enfants de $\mathrm{C}$ '. Donc, l'individu $\mathrm{C}$ est l'arrière-grand-père des maisonnées précitées. Cela dit, $\mathrm{A}$ et $\mathrm{B}$ peuvent décider de se nommer d'après $\mathrm{D}$, le père de $\mathrm{C}$, surtout si $\mathrm{D}$ n'a eu qu'un seul fils $\mathrm{C}$ et que $\mathrm{A}$ et $\mathrm{B}$ veulent perpétuer son nom. Il ne semble donc pas y avoir une règle précise pour cela.

25 Voir Osu (2000b) pour une étude de la négation en ikwere et Osu (2010), en particulier, pour la réduplication. 
Ainsi, la lignée Ofu [òfú], ${ }^{26}$ fait partie d'un groupement de lignées ḿḅú qui s'appelle ńhÊmménòbádờ. Le terme ḿbú est souvent traduit en anglais par 'compound' et en français par 'concession' ou 'campus'. C'est à mon avis un emploi métonymique puisque 'compound' ou 'concession, campus' désigne les enclos familiaux de l'époque ancienne. Quant à ḿḅú

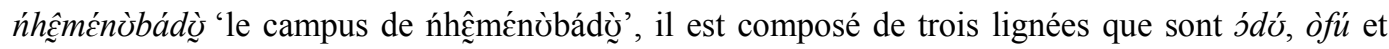
èlè $k^{w} a ̀ c \hat{\imath}$. L'espace ḿbú s'insère à son tour dans un groupement de ḿḅú, c'est-à-dire j̀hà 'village, commune, ville, pays'. Le terme j̀hà s'applique donc à tout groupement au-delà de la taille du campus. Plus précisément, dans l'entrée ḿbú, on lira dans le champ «notes et commentaires » l'information suivante :

ḿbú est un groupement de lignées souvent traduit en anglais par 'compound' et en français par 'concession' ou 'campus'. C'est à mon avis un emploi métonymique puisque 'compound' ou 'concession, campus' désigne les enclos familiaux de l'époque ancienne. L'espace ḿmbú s'insère à son tour dans un groupement de ḿbúu, c'est-à-dire j̀hà 'village, commune, ville, pays'. Le terme jhà s'applique donc à tout groupement au-delà de la taille du campus.

La dénomination d'un groupement de personnes, de maisonnées, d'un village, d'une ville ou d'un pays peut toutefois recourir à d'autres moyens. L'un de ces moyens consiste à prendre le nom d'un élément de la nature, par exemple celui d'un arbre célèbre. Ainsi, d'après les récits oraux transmis de génération en génération, le nom Ogbakiri [òbàkìrì] (la commune d'origine du présent auteur) serait une déformation de ṁbàkìrì 'arbre à semelles' (Pentaclethra macrophylla). ${ }^{27}$ Lorsque les premiers Européens sont arrivés dans la commune, ils auraient vu cet arbre en plein centre du village et auraient demandé son nom. Cet arbre aurait servi comme repère et graduellement serait devenu un moyen d'identifier la commune dans laquelle il se trouvait. L'histoire raconte également que n'étant pas capables de réaliser la nasale syllabique représentée par $m$ selon les conventions de l'orthographe, ces Européens ont réalisé et noté $o$ à la place, ce qui a donné Ogbakiri.

3.4 Calendrier. Illustrons avec l'entrée èké :

Dans le calendrier ikwere, èké occupe une place à part car bien que désignant un jour, il n'occupe pas pour autant un rang spécifique dans l'ordre des jours de la semaine. Il n'est pas l'un des cinq jours de la semaine ikwere mais revient systématiquement tous les quatre jours. Cela signifie donc qu'il peut tomber n'importe lequel des cinq jours. Par exemple, il peut tomber un jour de ryábó ou d'èbèrì ou encore d'áhyá 'bárámá c̀lì (voir plus loin pour la traduction de ces termes). Il est admis, de façon générale dans la tradition ikwere, que les esprits qui veillent sur les communes arrivent des quatre coins du monde. Ces esprits se réunissent tous les quatre jours pour échanger des informations et faire le point sur leurs activités. On croit également que ces esprits pénètrent dans les communes en traversant les rivières, et c'est pourquoi il est fortement déconseillé, voire

26 C'est le nom de famille du présent auteur, dans le dialecte d'Ogbakiri. Il a pour variante Osu qu'on rencontre dans d'autres dialectes mais aussi en igbo. Les premiers membres de la famille à être scolarisés dans la première moitié du vingtième siècle ont adopté cette variante pour se rapprocher de l'igbo qui était la langue dominante dans la région aussi bien dans l'administration, à l'église qu'à l'école. C'est pourquoi aujourd'hui encore les deux formes coexistent: dans le village la famille est connue sous le nom d'Ofu tandis que ceux qui sont scolarisés à l'école utilisent $O s u$.

27 Burkill $(1995: 252)$ 
interdit aux pêcheurs de boucher le passage des rivières en un jour d'èké. La tradition orale rapporte que certains pêcheurs qui n'ont pas respecté cet interdit dans le passé ont vu leurs filets de pêche détruits. Cette information se trouve dans le champ ng1 dans l'entrée èké.

Voici les cinq jours de la semaine, repris, publié et distribué depuis les années 2000 par M. Albert P. Okpara du village de Rumoru-Ogbakiri (Rivers State, Nigeria) :

\begin{tabular}{|c|c|c|}
\hline Transcription phonétique & $\begin{array}{l}\text { Transcription } \\
\text { phonologique }\end{array}$ & Orthographe ikwere \\
\hline Premier jour : áhyá'ḅárámá c̀lì & áhyá’ḅáráḅá èlì & áhịakpárámá ẹ̀lì \\
\hline Deuxième jour : èbèrì & èbèrì & èbèrì \\
\hline Troisième jour : ḿmákàrà & ḿḅákàrà & ḿmákàrà \\
\hline Quatrième jour: ńním & ńlínụú & ńním \\
\hline Cinquième jour : ryáḅó & ryábóó & rịágbó \\
\hline
\end{tabular}

Dans la commune d'Ogbakiri, le ryáḅó est une journée sainte et donc un jour férié. Il est interdit d'aller aux champs pour effectuer un travail difficile tel que sarcler. On peut néanmoins s'y rendre si c'est pour récolter des racines de manioc ou encore des légumes pour le repas. Ceux qui produisent du vin de palme le jour du ryábó n'ont pas le droit de le vendre : ils l'apportent directement à la salle de réunion du village pour une consommation collective. Le chef suprême du village doit s'assurer que les ingrédients élémentaires tels que le sel, le piment, l'huile, le poisson fumé, la viande nécessaires pour préparer le plat avec lequel on consomme le vin de palme sont disponibles.

Tout décès qui survient le jour de ryábó est hautement craint, car c'est un très mauvais signe pour la famille et pour toute la commune. On trouve cette information dans l'entrée ryábó dans le champ ng1 comme illustrée en annexe.

3.4 Mariage. Illustrons le mariage avec l'entrée órá ótibi 'réclusion prénuptiale'. Cette expression est composée de deux unités nominales :

órá : réclusion

ótìbi : ce terme fait référence au cycle menstruel de la femme.

Ainsi, une femme peut dire :

(7) ̀̀ zîm nótibì.
m Zî-m
nú-ótìì̀
1SG être.LOC-ACCORD 1SG REL-cycle menstruel

Je suis dans ma période menstruelle (j'ai mes règles). 
Et l'on peut dire d'une jeune fille :

(8)

$\begin{array}{llll}\text { ò } & \text { nèmé órá ótibì. } & & \\ \text { ò } & \text { nèmé } & \text { órá } & \text { ótìbì } \\ 3 \text { 3SG } & \text { INACC-faire } & \text { réclusion } & \text { menstruation }\end{array}$

Elle fait sa réclusion prénuptiale.

Après un traitement linguistique (transcription phonétique et phonologique, découpage morphologique, identification de la partie du discours, schème tonal, etc.), la graphie de l'entrée et des exemples illustratifs, suit alors dans le champ «note générale» un commentaire sur la réclusion prénuptiale et le mariage chez les Ikwere.

En effet, la réclusion prénuptiale est une étape dans le processus du mariage. Il faut noter que l'endogamie (mariage au sein de sa communauté) et l'exogamie (mariage en dehors de sa communauté) sont toutes les deux permises et pratiquées chez les Ikwere.

De façon générale, le choix de la jeune fille à épouser fait l'objet d'un consensus entre les parents et le garçon. En tout état de cause, le garçon ne s'opposera pas au choix des parents car, de par leur expérience, ils savent quelles familles donnent une bonne éducation à leurs enfants. Après tout, ne dit-on pas chez les Ikwere que l'arbre le plus haut voit le plus loin ? Accompagnés par quelques membres de leur famille, portant des cruches de vin de palme et autres boissons avec eux, le père et le garçon se présentent dans la famille de la jeune fille qui est censée être $\grave{m} b e ̀ l e ̀ k^{w} e ́$ 'jeune fille nubile, non mariée'.

C'est une visite de courtoisie, appelée $a \grave{a} k^{w} a \check{a}$ ákâ n-j́rj̀ 'frapper à la porte', pendant laquelle ils dévoilent leur intention à la famille de la jeune fille. Si l'accueil est positif et que la jeune fille partage l'envie de se marier avec le garçon, un autre rendez-vous est programmé pour énumérer au garçon et aux siens la longue liste de choses qu'ils doivent apporter à chaque membre de la famille de la jeune fille. Cette liste est préparée avec l'aide des parents de celle-ci. En outre, étant entendu que la jeune fille, comme tout enfant ikwere, n'appartient pas qu'à ses parents biologiques mais à toute sa lignée, le garçon et sa famille doivent obligatoirement rendre visite à certains membres emblématiques de la famille de la fille s'ils veulent que le mariage soit célébré sans souci.

Ensuite, le garçon apporte une dot j̇k ơ íwáy rísî ńwèrè 'le fait de payer l'argent de la tête de l'épouse', qui aura fait l'objet d'une négociation. En réalité, la dot comprend diverses choses qui vont de l'argent à des vêtements en passant par des services à rendre à la grand-mère de la fille, par exemple. Ainsi, le garçon pourra employer l'expression àgâ izể ńwèrè̀ pour dire qu'il fait les démarches auprès de la famille de sa future femme afin d'obtenir l'autorisation de l'épouser.

A l'issue de ces différentes visites, vient le jour de la cérémonie appelée j̀wõ máyâ rísî ńwèrè 'boire le vin de la tête de l'épouse' ou 'boire en l'honneur de la mariée'. C'est une occasion pour les deux familles de se réunir et de proclamer les deux époux mariés. Le processus de mariage vu du côté de la famille de la fille se dit c̀ní ńzí faisant référence au fait de donner la fille en mariage. La fille emploiera le verbe j̣̀j́ ńzî 'prendre époux', tandis que le garçon dira j̀ló ńwèrè 'prendre épouse'. Remarquons que pour le même acte, le garçon et la fille n'emploient pas le même verbe. Linguistiquement, non seulement la base verbale est différente pour les deux cas (garçon et fille) mais le complément aussi. La langue ikwere semble donc indiquer que la réalité du mariage pour l'un ou l'autre conjoint n'est pas la même et n'a pas les mêmes implications sociales. En somme, se marier n'est pas le même acte pour le garçon ou pour la fille. 
C'est alors qu'intervient la réclusion prénuptiale. ${ }^{28}$ Aidée par sa mère, la future mariée reçoit pendant 4 à 8 jours $^{29}$ ses amies, ses cousines matrilatérales et patrilatérales ainsi que les filles de la famille du mari, qu'elle aura soigneusement choisies. Ces filles s'occupent d'elle et lui tiennent compagnie. Ensemble, elles se font belles, elles aussi ; et peut-être pour se prémunir à la fois contre les mauvais esprits et certaines maladies de peau d'origine parasitaire (cf. Burkill 1995 : 293), elles se badigeonnent d'une pâte tirée de la plante ihyé 'bois rouge, bois de cam' (Baphia nitida Lodd ou Baphia pubescens Hook ${ }^{30}$ qu'elles auront produite après avoir écrasé et moulu le bois des heures durant au moyen de pierres. Elles chantent, dansent, préparent à manger, puis mangent ensemble. La nourriture typique est une sauce à base de feuilles de la plante àjà (Afzelia bipindensis Harms). A la nuit tombante, les garçons entrent et les cherchent, souvent dans le noir (lumières éteintes), pour leur mettre la main aux fesses. Cela s'appelle àbárí ikóró 'jouer à la jeunesse'. C'est l'information contenue dans ce dernier paragraphe qui se trouve dans le champ « ng1 \de l'entrée órá ótibì comme illustré en annexe.

A la fin de la réclusion, la jeune fille, conduite par les filles et les femmes qui sont autorisées à l'accompagner, quitte sa famille d'origine pour aller s'installer dans la famille de son époux, d'où l'expression verbale àlâ ̀̀zî 'partir (dans la famille de son) époux', car la résidence chez les Ikwere est patrilocale. Si pour une raison quelconque le garçon part s'installer dans la famille de son épouse, cela est vu comme une preuve de faiblesse de sa part, et l'on peut se moquer de lui, on peut l'appeler « l'épouse de sa femme ». Plus sérieusement, on considère que c'est un malheur qui s'abat sur la famille du garçon et dont il faut chercher la cause. La fille emporte aussi des biens que sa famille d'origine lui offre en cadeaux de mariage : tabouret en bois, ustensiles de cuisine, une malle remplie d'habits, etc.

Une fois mariée, la jeune fille devient ńwèrè 'épouse'. Dans sa nouvelle famille, on la désignera du nom dérivé òrí $\grave{\varepsilon} k^{w} \grave{d}$, composé du préfixe agentif $\dot{o}-+$ la base verbale rí (évoquant l'idée de manger) + le complément $\grave{\varepsilon} k^{w} \grave{\partial}$ 'biens', l'ensemble de l'expression signifiant : consommatrice des biens de son mari, et par extension, de la belle-famille. Car, dans la conception ikwere, c'est désormais pour le bien-être de son épouse que doit vivre et travailler le mari ; on semble ainsi tenir peu compte des travaux quotidiens qu'elle accomplit.

Pour autant, si la femme intègre la lignée de son mari, elle ne renonce pas à son appartenance à sa famille d'origine. Ainsi, elle n'est pas obligée de changer son patronyme pour celui de son mari. L'époux, ̀̀zî, est le point stable du couple (l'épouse gardant toujours le droit de repartir dans sa famille de naissance, en cas de litige ou de divorce) et, à ce titre, il est seul apte à représenter la famille, d'abord au sein de la branche familiale, puis au sein du village, puis en dehors, s'il y a lieu. Il est le gendre de la famille de son épouse et y est appelé j́g̀̀ (anglais : in-law). Il les appelle aussi ógj̀. Entre sa famille et celle de son épouse existe désormais une relation de ógj̀ 'bellefamille'. En effet, en fonction du contexte, ce terme peut désigner les beaux-parents, la bellefamille ou le gendre.

En cas de litige dans le couple, par exemple une dispute conjugale, la fille peut décider de quitter sa nouvelle famille pour réintégrer, ne serait-ce que pour une courte période, sa famille de naissance. On dit d'elle :

28 Ce qualificatif est employé ici faute de mieux. Comme je le décris plus loin, le mariage a officiellement été célébré. J'ignore si une telle réclusion a un rapport quelconque avec la consommation du mariage.

29 Les Anciens du village racontent que cela durait beaucoup plus longtemps dans l'ancien temps.

30 Le mot íhyé qui désigne aussi la couleur rouge en ikwere est vraisemblablement issu du nom de ce bois. 
(9)

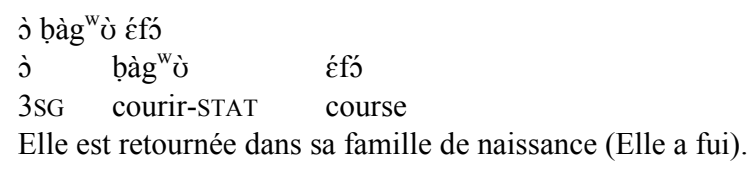

Selon Ogundu (1981), on distingue trois types de mariage chez les Ikwere: polygamie, monogamie et lévirat. La polygamie est une pratique ancienne qui se fait de plus en plus rare. Mais lorsqu'elle est pratiquée, la première femme est désignée comme óbòtù. Sauf cas de divorce et de remariage, sauf aussi cas d'adultère et d'infidélité, jamais de la mémoire de ma grand-mère on n'a vu une femme mariée à deux hommes en même temps. Autant la polygamie paraît normale, autant une relation extra-conjugale est réprouvée. La personne a vite fait de devenir l'objet de divulgation publique, notamment par le club òjée spécialisé dans ce genre de tâche d'intérêt commun.

Le lévirat, ńwèrè ḿm 'bé 'épouse héritée', est également une pratique ancienne. Il est toutefois intéressant de noter que si les femmes sont ainsi reprises par les frères de leurs maris défunts, les veufs ne sont pas repris par les sœurs de leurs épouses décédées.

\section{Conclusion}

J'ai voulu, à travers ces quelques exemples, montrer comment j'exploite les travaux que je mène dans mes diverses activités de recherche pour mon projet de dictionnaire. Pour chacune des entrées, j'essaie de renseigner les divers champs prévus. J'essaie également de mettre en lumière la culture dans laquelle s'inscrit la langue objet de mes recherches. Ainsi, après le traitement linguistique d'une entrée, je passe par le champ «note générale » pour fournir les renseignements culturels chaque fois que cela s'avère utile. Dans le cas des noms de personne par exemple, j'introduis le nom, puis je rappelle l'énoncé dont il est extrait, je propose un découpage morphologique, puis une traduction mot à mot, et enfin une traduction en français et en anglais, sans oublier les circonstances qui entourent le choix ou la création de ce nom. Et c'est là l'aspect culturel, car ces circonstances tendent à marquer la spécificité de la culture ikwere, sans pour autant prétendre que le peuple Ikwere est le seul à procéder ainsi. Je décris également les mécanismes linguistiques qui participent de la construction du nom et du sens qui lui est associé.

\section{Références}

Akin, Salih. (dir.) 1999. Noms et re-noms : la dénomination des personnes, des populations, des langues et des territoires. Rouen, Publications de l'Université de Rouen.

Alerechi, Roseline I.C. 2007a. A dialect survey of Ikwere: a phonological perspective. Unpublished PhD dissertation. Port-Harcourt, University of Port-Harcourt.

Alerechi, Roseline I.C. 2007b. Labial variation in Ikwere. In Ozo-Mekuri, N. (ed.), Convergence: English and Nigerian languages: a festschrift for Munzali, A. Jibril. Port-Harcourt (Nigeria), M \& J Grand Orbit Communications. 347-369. 
Alerechi, Roseline I.C. 2008. Vowel variation in Ikwere. In Ozo-Mekuri, N., Udoh, I.I.L., Anyanwu, O. (eds.), Critical issues in the study of Linguistics, Languages and Literatures in Nigeria: a festschrift for Conrad Max Benedict Brann. Port-Harcourt (Nigeria), M \& J Grand Orbit Communications. 297-330.

Alerechi, Roseline I.C., Weje, Annette U. 2013. The counting system in Ikwere. In Ozo-Mekuri, N. \& Chan, E. S. L. (eds.), The numeral systems of Nigerian languages. Port-Harcourt (Nigeria), M \& J Grand Orbit Communications. 175-187.

Asante, Molefi K. 1991. The book of African names. Trenton (N.J.), Africa World Press.

Béjoint, Henri, Thoiron, Philippe. (éds.). 1996. Les dictionnaires bilingues. Louvain-la-Neuve, Duculot. Bouquiaux, Luc. 2002. Le dictionnaire thématique birom, Nigeria. In Colombel, V. de \& Tersis, N. (éds.), Lexique et motivation : perspectives ethnolinguistiques. Paris, Peeters. 17-24.

Burkill, Humphrey M. 1995. The useful plants of West Tropical Africa. Vol. 3: Families J-L. Kew, The Trustees of Royal Botanic Gardens.

Caron, Bernard, Amfani, Ahmed H. 1997. Dictionnaire français-haoussa suivi d'un index haoussa-français. Paris, Karthala.

Choi-Jonin, Injoo, Delhay, Corinne. 1998. Introduction à la méthodologie en linguistique : application au français contemporain. Strasbourg, Presses Universitaires de Strasbourg.

Clements, George N., Osu, Sylvester N. 2005. Nasal harmony in Ikwere, a language with no phonemic nasal consonants. Journal of African Languages and Linguistics 26.2. 165-200.

Clements, George N., Osu, Sylvester N. 2002. Explosives, implosives, and nonexplosives: the linguistic function of air pressure differences in stop. In Gussenhoven, C. \& Warner, N. (eds.), Laboratory phonology 7. Berlin, Mouton de Gruyter. 299-350.

Cristinoï, Antonia, Nemo, François. 2013. Challenges in endangered language lexicography. In Kwary, D.A., Wulan, N. \& Musyahda, L. (eds.), Lexicography and dictionaries in the information age: selected papers from the 8th ASIALEX international conference, Bali 20-22 August 2013. Denpassar, Airlangga University Press. 126-132.

Dikki-Kidiri, Marcel, Mbodj, Chérif, Edema, Atibakwa B. 1997. Des lexiques en langues africaines (sängo, wolof, lingala) pour l'utilisateur de l'ordinateur. Meta : journal des traducteurs/Meta: Translator's Journal 42.1. 94-109.

Donwa-Ifode, Shirley O., Ekwulo, Samuel A. 1987. Ikwere orthography. In Orthographies of Nigerian Languages: manual (version remaniée par R.N. Agheyisi). Lagos, National Language Centre, Federal Ministry of Education.

Dotoli, Giovanni. 2008. La construction du sens dans le dictionnaire. Paris, Hermann.

Echeruo, Michael J.C. 1998. Igbo-English dictionary: a comprehensive dictionary of the Igbo language, with an English-Igbo index. New Haven, London, Yale University Press.

Ekwulo, Samuel A. 1970. Ikwerre mbom.-Ed. rev.-1981.

Ekwulo, Samuel A. 1975. (ed.). Elulu ikwere = ikwere proverbs: in ikwere and English. In: Rivers Bilingual Series no.1, Port-Harcourt (Nigeria), Rivers State Council for Arts and Culture.

Emenanjo, Emmanuel N. 1978. Elements of modern Igbo grammar: a descriptive approach. Ibadan (Nigeria), Oxford University Press.

Enyia, Tony 2008. Speaking Ikwere: a beginners' first year course for language student (Book 1: Intermediate). Port Harcourt (Nigeria), Assubless Publishers. 
Grundy, Valerie. 1996. L'utilisation d'un corpus dans la rédaction du dictionnaire bilingue. In Béjoint, H. \& Thoiron, P. (éds.), Les dictionnaires bilingues. Louvain-la-Neuve, Duculot. 127149.

Kropp-Dakubu, Mary Esther. 1999. Ga-English dictionary with English-Ga index. Accra (Ghana), University of Ghana.

Leguy, Cécile, Lebarbier, Micheline. 2006. (coord.). Des noms et des personnes. Cahiers de Littérature Orale 59-60 (Publications Langues'O).

Lemaréchal, Alain. 1989. Les parties du discours : sémantique et syntaxe. Paris, Presses Universitaires de France.

Marello, Carla. 1996. Les différents types de dictionnaires bilingues. In Béjoint, H. \& Thoiron, P. (éds.), Les dictionnaires bilingues. Louvain-la-Neuve, Duculot. 31-52.

Negga, Delombera. 2008. Approche linguistique des noms propres amhariques. Communication orale présentée à la Journée d'études éthiopiennes du 31 janvier 2008, Inalco - Département Afrique.

Ogundu, Joseph E. 1981. Life in Rumuoro Community in the Past and in the Present. Long Essay (in partial fulfilment of the requirements for the award of the associateship certificate in education). Port Harcourt (Nigeria), Rivers State College of Education (Institute of education, University of Ibadan, Nigeria).

Osu, Sylvester N. (à paraître). Le /-m/ final du verbe et l'accord minimum en personne en ikwere, une langue sans système d'accord. In Collins, C. (éd.), Accord-non accord. Travaux linguistiques du Cerlico 3 . Rennes, Presses Universitaires de Rennes.

Osu, Sylvester N. 2010. Entre réduplication et répétition en ikwere : de l'identification à l'identité. In Osu, S. N., Col, G., Garric, N., Toupin, F. (éds.), Construction d'identité et processus d'identification. Berne (Suisse), Peter Lang. 545-567.

Osu, Sylvester N. 2003a. Le morphème nu en ikwere. In Robert, S. (éd.), Perspectives synchroniques sur la grammaticalisation : polysémie, transcatégorialité et echelle syntaxiques. Paris, Peeters. 203-2015.

Osu, Sylvester N. 2003b. Semantic invariance, locating process and alterity: a TOPE-based analysis of the verbal prefix z- in Ikwere. Journal of Linguistics 39.3, 521-574.

Osu, Sylvester N. 2000a. La focalisation en ikwere. In Caron, B. (éd.) Topicalisation et focalisation dans les langues africaines. Louvain, Paris, Peeters. 209-227.

Osu, Sylvester N. 2000b. Negation marking in Ikwere. Linguistique Africaine 22, 57-106.

Osu, Sylvester, Clements, George Nick. 2009. Les nasals en ikwere. CORELA 7.

Platon. 1950. Le Sophiste. Euvres complètes II. Traduction nouvelle et notes par Léon Robin avec la collaboration de M.-J. Moreau. Paris, Gallimard. 216-338.

Roulon-Doko, Paulette. 2008. Dictionnaire gbaya-français : République Centrafricaine, suivi d'un dictionnaire des noms propres et d'un index français-gbaya. Paris, Karthala.

Rey-Debove, Josette, Rey, Alain. 2007. Préface. Le Nouveau Petit Robert. Dictionnaire alphabétique de la langue française, nouvelle édition du Petit Robert de Paul Robert, texte remanié et amplifié sous la direction de Rey-Debove, J. \& Rey, A. Paris, Le Robert. IX-XLII.

Sachnine, Michka. 1997. Dictionnaire yoruba-français suivi d'un index français-yoruba. Paris, Karthala.

Stewart, Julia. 1996. 1,001 African names: first and last names from the African continent. Secaucus (N.J.), Carol Publishing Group. 
Taylor, Frank William. 1995. Fulani-English dictionary. New York, Hippocrene Books. (First published 1932, OUP).

Tiérou, Alphonse. 1977. Le nom africain ou langage des traditions. Paris, G.-P. Maisonneuve et Larose.

Ugochukwu, Françoise, Okafor, Peter. 2004. Dictionnaire igbo-français suivi d'un index françaisigbo. Paris, Karthala.

Weje, Annette U. 2007. Compound verbs in Ikwere. Unpublished PhD dissertation. Port-Harcourt (Nigeria), University of Port-Harcourt.

Weje, Annette U. 2003. The logophoric pronoun in Ikwere. In Ozo-Mekuri, N. (ed.), Four decades in the study of language and linguistics in Nigeria: a festschrift for Kay Williamson. Aba (Nigeria), NINLAN. 748-751.

Williamson, Kay, Ekwulo, Samuel A., Alerechi, Roseline I.C., Wali, Jude T.N., Weje, Annette. 2010. Reading and writing Ikwere. Port-Harcourt (Nigeria), M\&J Grand Orbit Communications.

Williamson, Kay. 1972. Igbo-English dictionary. Benin-City (Nigeria), Ethiope.

Williamson, Kay 1970. Reading and writing Ikwere. Ibadan (Nigeria), University of Ibadan.

\section{Annexe}

Ci-dessous, des extraits de la base de données électronique du projet de dictionnaire ikwere-

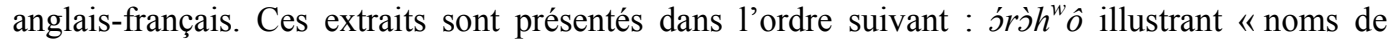
personne », ryábó illustrant «calendrier», óráa ótìbi pour «mariage », et enfin, nu pour « caractérisation métalinguistique des unités ». Il convient toutefois de rappeler que le travail sur le projet de dictionnaire est en cours. 
lex lexeme (phonétique)

\phm phonological transcription

Imb morpheme boundary

lortho Ikwere official orthography

Isnd native speaker's recorded pronunciation

Itp tone pattern

Itcl tone class

Iss syllable structure

Ivform uninflected verb form

Ips part of speech

Igen gender

Inum number

Imorph morphological composition

legnc English gloss nominal complement

ffgc French gloss nominal complement

Igik gloss in Ikwere

lpkw part of speech in Ikwere

Eglitt literal meaning of complement in English

lit2 literal meaning of complement in French

Igleng gloss in English of lexeme

Igfr gloss in French of lexeme

leg1 example 1

lwwgloss 1 word for word gloss 1

lengloss English gloss for examnle 1

Ifrgloss French gloss for example 1

leg2 example 2

Iwwtrans word for word translation for examnle lengtrans English translation for examnle

Ifrtrans French translation for example

ldd *

lcomvar comments on variants

Idv dialect of variant

thom homonym of lexeme

- Isyn synonym of lexeme

lant antonym

Idfl meaning 1

Idf2 meaning 2

Idf3 meaning 3

- Irenvoi cross reference

letym etymology

lorig source language of lexeme

- Imchar metalinguistic characterisation of lexem

- Idefn definition of lexeme

Ipix picture $\operatorname{sroh}^{\mathrm{w}} \mathrm{o}$

oroh wo

o-ro-hw $\mathrm{u}-\mathrm{o}$

ọrowhuo

[HLF]

vevev

n

préfixe nominal+racine+base verbale+support du ton de la negation

The family will not disappear

La maisonnée ne disparaîtra pas

brò $\mathrm{h}^{\mathrm{w}} \mathrm{\delta} \quad \partial \mathrm{h}^{\mathrm{w}} \mathrm{u}$

house disappeartneg disappear

Our family will not disappear.

Notre famille ne disparaîtra pas. 
Itabl table 1

Itab2 table 2

inph phonology notes

ing1 general notes and comments 1

lng2 general notes and comments

linf1 informant 1

linf2 informant 2

linf3 informant 3

linfsex sex of informant

lage age of informant

linftown town of informant

Irdd date of recording
Après plusieurs accouchements successifs, les parents n'ont que des filles. L'arrivée d'un garçon est source de soulagement. En effet, alors même que les filles sont censées quitter leur famille d'origine pour aller s'installer dans la famille de leur mari, les garçons, eux, restent dans la famille; ils sont donc les gages de la continuité de la famille. On note l'emploi réduplicatif du verbe "disparaître". On note aussi la négation signalée par le ton descendant sur la voyelle finale de la base verbale. Cette structure permet au locuteur d'asserter contre autrui, réel ou imaginaire, que la famille, la sienne est appelée à se perpétuer. A travers la première mention de < disparaître>, il introduit une occurrence du domaine notionnel de celui-ci, et le présente comme plus ou moins susceptible de se réaliser. Or, l'introduction de la copie et la juxtaposition lui permettent de rapporter cette première occurrence à l'occurrence représentative du domaine $<$ disparaitre>. Le domaine se présente cette fois comme stabilisé, interprété : si on considère ce qu'on appelle vraiment <disparaître>, l'arrivée d'un garçon fait que la famille pour l'éviter.

nom de personne 


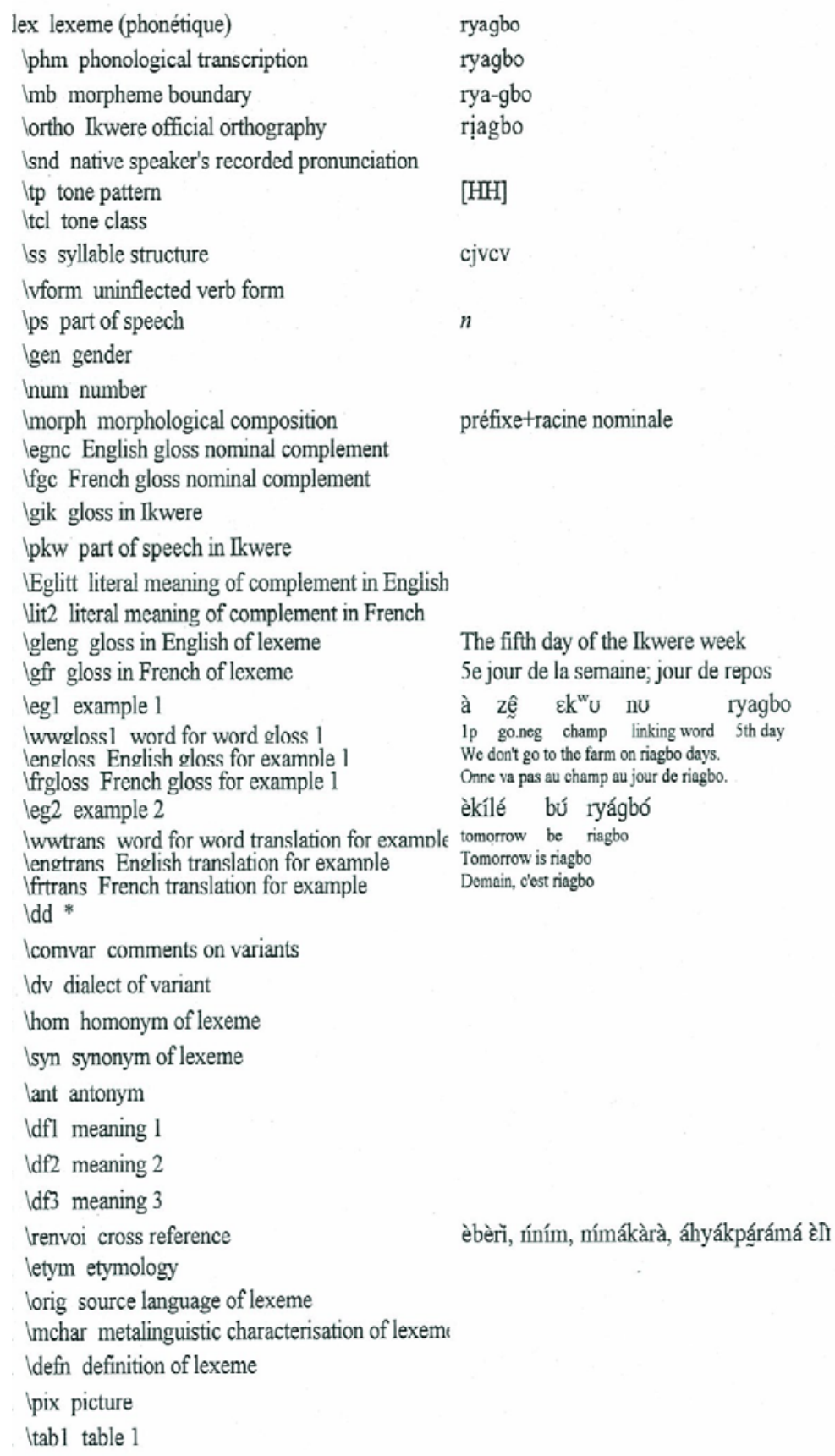


Itab2 table 2

Inph phonology notes

lngl general notes and comments 1
Dans le calendrier ikwere, ekne occupe une place à part car bien que désignant un jour, il n'occupe pas pour autant un rang spécifique dans l'ordre des jours de la semaine. Il n'est pas l'un des cinq jours de la semaine ikwere mais revient systématiquement tous les quatre jours. Cela signifie donc qu'il peut tomber à n'importe quel des cinq jours. Par exemple, il peut tomber un jour de riagbo ou d' eberi ou encore d'ahiakpnarama eli. Il est admis de façon générale dans la tradition ikwere que les esprits qui veillent sur les communes arrivent des quatre coins du monde. Ces esprits se réunissent tous les quatre jours pour échanger des informations et faire le point sur leurs activités. On croit également que ces esprits pénètrent dans les communes en traversant les rivières, et c'est pourquoi il est fortement déconseillé, voire même interdit aux pêcheurs de boucher le passage des rivières en un jour d'ekne. La tradition orale rapporte que certains pêcheurs qui n'ont pas respecté cet interdit dans le passé ont vu leurs filets de pêches détruits. Dans la commune d'Ogbakiri, le riagbo est une journée sainte et donc un jour férié. Il est interdit d'aller au champ pour effectuer un travail difficile tel que sarcler. On peut néanmoins s'y rendre si c'est pour récolter des racines de manioc ou encore des légumes pour le repas. Ceux qui produisent du vin de palme le jour du riagbo n'ont pas le droit de le vendre: ils l'apportent directement à la salle de réunion du village pour une consommation collective, à charge au chef suprême du village de s'assurer que les ingrédients élémentaires tels que le sel, le piment, l'huile, le poisson fumé, la viande nécessaires pour préparer le plat avec lequel on consomme le vin de palme sont disponibles. Tout décès qui survient à un jour de riagbo est hautement craint car c'est un très mauvais signe pour la famille et pour toute la commune.
Ing2 general notes and comments

linfl informant 1

inf2 informant 2

linf3 informant 3

linfsex sex of informant

lage age of informant

linftown town of informant

lrdd date of recording 


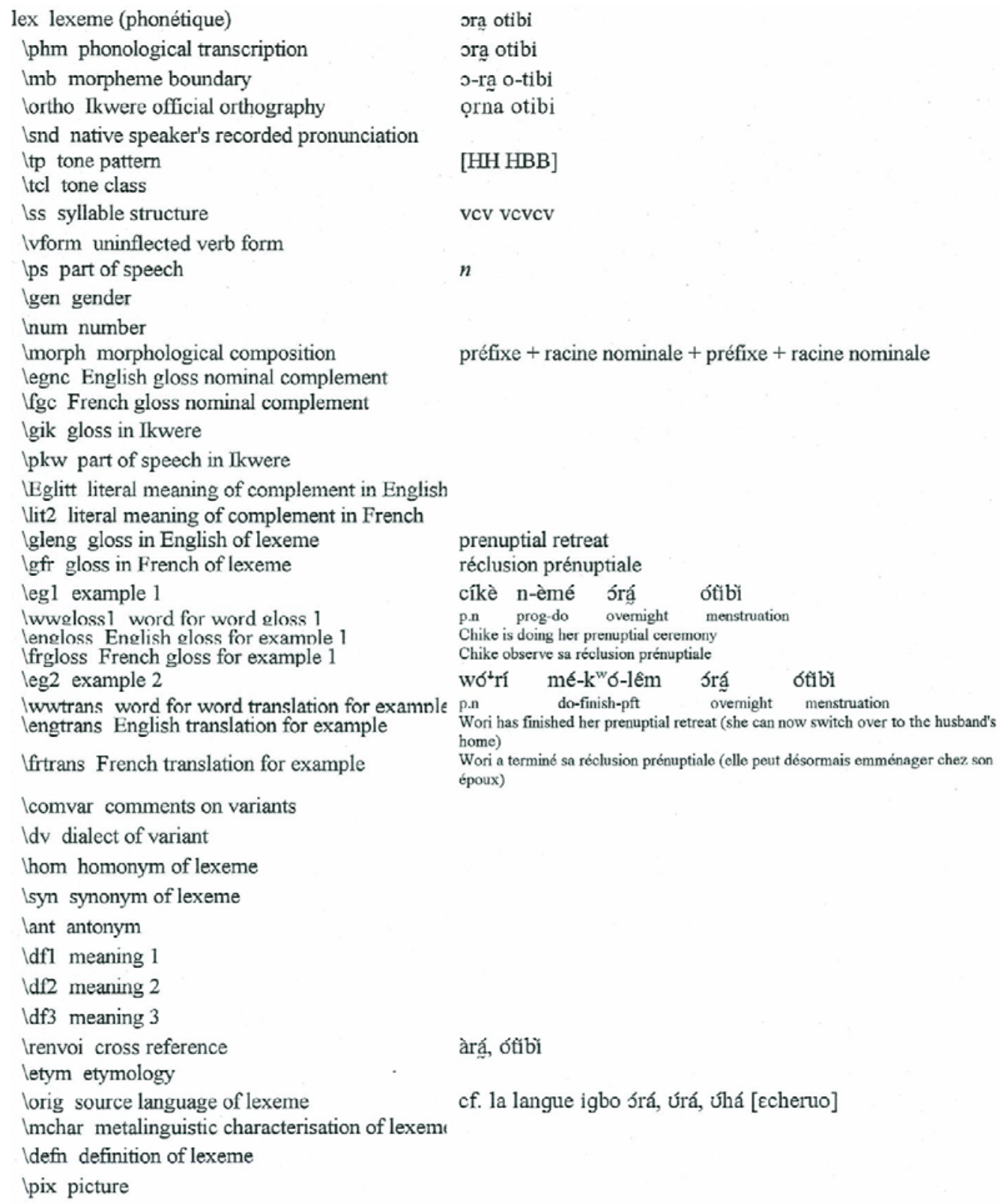


Itabl table 1

Itab2 table 2

Inph phonology notes

lng1 general notes and comments 1
La réclusion prénuptiale est une étape dans le processus du mariage. Aidée par sa mère, la future mariée, reçoit pendant 4 à 8 jours ses amies, cousines matrilatérales et patrilatérales ainsi que les filles de la famille du mari, qu'elle aura soigneusement triées. Ces filles s'occupent d'elle et lui tiennent compagnie. Ensemble, elles se font belle, elles aussi ; et, peut-être pour se prémunir à la fois contre les mauvais esprits et certaines maladies de peau d'origine parasitaire (cf. Burkill 1995 : 293), elles se badigeonnent avec la pâte tirée de la plante ihye, "bois rouge, bois de cam (Baphia nitida Lodd ou Baphia pubescens Hook)" qu'elles auront produite après avoir écrasé et moulu le bois des heures durant au moyen de pierres ; elles chantent, dansent, préparent à manger, puis elles mangent ensemble. La nourriture typique est une sauce à base des feuilles de la plante aja (Afzelia bipindensis Harms) afin peut-être de se prémunir contre la constipation. A la nuit tombante, les garçons arrivent et les cherchent, souvent dans le noir (les lumières éteintes), visant en tout et pour tout à leur mettre la main aux fesses. Cela s'appelle agbari ikoro "jouer à la jeunesse".

lng2 general notes and comments

linfl informant 1

linf2 informant 2

linf3 informant 3

infsex sex of informant

lage age of informant

linftown town of informant

lrdd date of recording 
lex lexeme (phonétique)

Iphm phonological transcription

Imb morpheme boundary

lortho Ikwere official orthography

Isnd native speaker's recorded pronunciation

Itp tone pattern

Itcl tone class

Iss syllable structure

Ivform uninflected verb form

lps part of speech

Igen gender

lnum number

Imorph morphological composition

legnc English gloss nominal complement

lfgc French gloss nominal complement

Igik gloss in Ikwere

Ipkw part of speech in Ikwere

Eglitt literal meaning of complement in English

lit2 literal meaning of complement in French

Igleng gloss in English of lexeme

Igfr gloss in French of lexeme

legl example 1

lwwgloss 1 word for word gloss 1

lengloss English gloss for examnle

Ifrgloss French gloss for example 1

leg2 example 2

wwtrans word for word translation for examnls

lengtrans English translation for examnle

frtrans French translation for example

lcomvar comments on variants

Idv dialect of variant

thom homonym of lexeme

Isyn synonym of lexeme

lant antonym

Idfl meaning 1

Idf2 meaning 2

Idf3 meaning 3

Irenvoi cross reference

letym etymology

lorig source language of lexeme

Imchar metalinguistic characterisation of lexemı Etant donné E1 (un premier terme, c'est-à-dire une situation, la valeur d'un chiffre, un instant, une relation prédicative, etc.), nu introduit E2 (un deuxième terme) et le fonde comme le terme à prendre en compte de façon à redélimiter El. linking word

relateur

ǹrǐ n-ótù

10 and-1

11

3 kà nư ŕwế lá-lêm ó

3sg say that logophoric go-pft sfp

He says that he has gone

Il dit quil est parti nu

nu

[L] or $[\mathrm{H}]$

cV

linking word

Idefn definition of lexeme 